Dermatology 2013;226:13-14

DOI: $10.1159 / 000345763$

\section{Facial Basal Cell Carcinomas Recurring after Imiquimod Therapy}

\section{A.M. Skaria}

Centre de Dermatochirurgie Vevey, Vevey, Switzerland

Key Words

Basal cell carcinoma $\cdot \operatorname{Imiquimod} \cdot$ Nonmelanoma skin cancer

I would like to add an observation to the article of Fiechter et al. [1]. With the increase in incidence of nonmelanoma skin cancer, taking charge of these patients is getting more and more challenging for the dermatologist and the healthcare system [2]. Some authors warn about major implications for future healthcare services [3]. Therefore it is only understandable that dermatologists long for simple solutions. One of these seemed the treatment of nodular or more aggressive basal cell carcinoma (BCC) with imiquimod $[4,5]$. Although there is no written consensus concerning imiquimod treatment today, the too optimistic indication of certain authors during the last few years, willingly supported by the industry, is not yet eradicated.

I have looked at the histology of all my patients treated since the beginning of 2012 with Mohs surgery. Eight patients had had previous imiquimod treatment for the operated lesion. Five lesions were biopsied before the treatment with imiquimod. Three patients were treated with imiquimod without prior biopsy (2 patients had clinically a nodular tumor according to the referring dermatologist, in 1 patient the type of BCC was not specified). The others were 2 actinic keratoses ( 1 with histological doubt, perhaps a superficial basosquamous carcinoma), 1 patient had superficial BCC and 2 patients had a nodular BCC (table 1).

The histological examination during Mohs surgery revealed 1 multifocal basosquamous carcinoma, 3 morpheaform, 1 infiltrative, 1 nodular and 1 solid BCC. This means that 7 out of 8 patients showed an increase in the aggressivity of their tumor. The major point of concern is that nodular BCCs and a doubtful basosquamous carcinoma were treated by imiquimod and that the delay after this treatment was up to 4 years with 5 out of these 8 cases seemingly clinically healed during this period.

In analogy to the article mentioned above, concern is raised whether imiquimod may select more aggressive tumor cells or may just convey a natural course of tumor recurrence as we see with other treatment modalities. In any way there should be a clear consensus today that imiquimod treatment should only be applied to superficial BCC which have been biopsied before. Treatment of nodular or more aggressive BCC with imiquimod is obsolete. In case of recurrence after imiquimod treatment, surgery is always more damaging and more expensive because of the increased aggressivity as well as the poor clinical delimitation of these tumors.

Table 1. Patients with imiquimod treatment before Mohs surgery in 2012

\begin{tabular}{|c|c|c|c|c|c|c|c|c|}
\hline $\begin{array}{l}\text { Patient/age/ } \\
\text { sex }\end{array}$ & $\begin{array}{l}\text { Local- } \\
\text { ization }\end{array}$ & $\begin{array}{l}\text { Primary diagnosis } \\
\text { (histology) }\end{array}$ & $\begin{array}{l}\text { Clinical } \\
\text { diagnosis }\end{array}$ & $\begin{array}{l}\text { Diagnosis at Mohs } \\
\text { (histology) }\end{array}$ & $\begin{array}{l}\text { Delay of } \\
\text { recurrence } \\
\text { after imiqui- } \\
\text { mod treatment }\end{array}$ & $\begin{array}{l}\text { Imiquimod } \\
\text { treatment, } \\
\text { weeks }\end{array}$ & $\begin{array}{l}\text { Treatment } \\
\text { scheme, } \\
\text { days/ } \\
7 \text { days }\end{array}$ & $\begin{array}{l}\text { After } \\
\text { imiquimod } \\
\text { clinically } \\
\text { healed }\end{array}$ \\
\hline $\mathrm{HH} / 64 / \mathrm{M}$ & temple & $\begin{array}{l}\text { actinic keratosis or superficial } \\
\text { basosquamous carcinoma }\end{array}$ & & $\begin{array}{l}\text { multifocal basosquamous } \\
\text { carcinoma }\end{array}$ & 1 year & 6 & $5 / 7$ & yes \\
\hline $\mathrm{JJ} / 44 / \mathrm{M}$ & cheek & actinic keratosis & & morpheaform BCC & 1 year & 6 & $5 / 7$ & yes \\
\hline $\mathrm{VR} / 70 / \mathrm{F}$ & nose & BCC nodular & & morpheaform BCC & 4 years & 6 & $5 / 7$ & yes \\
\hline $\mathrm{CJ} / 75 / \mathrm{M}$ & nose & BCC nodular & & morpheaform BCC & 2 years & 8 & $5 / 7$ & yes \\
\hline $\mathrm{PD} / 71 / \mathrm{F}$ & nose & no biopsy & nodular BCC & infiltrative BCC & 1 year & $3 \times 6-8$ & $5 / 7$ & no \\
\hline $\mathrm{AK} / 38 / \mathrm{F}$ & nose & superficial BCC & & solid BCC & 1.5 years & 4 & $5 / 7$ & yes \\
\hline $\mathrm{RS} / 64 / \mathrm{F}$ & shoulder & no biopsy & not specified & morpheaform BCC & 3 years & $3 \times 6$ & $5 / 7$ & no \\
\hline $\mathrm{FM} / 71 / \mathrm{F}$ & nose & no biopsy & nodular BCC & nodular BCC & 1 year & 6 & $5 / 7$ & no \\
\hline
\end{tabular}

\section{KARGER}

E-Mail karger@karger.com

www.karger.com/drm
(C) 2012 S. Karger AG, Basel

$1018-8665 / 13 / 2261-0013 \$ 38.00 / 0$
A.M. Skaria

Lecturer University of Bern

Centre de Dermatochirurgie Vevey

$\mathrm{CH}-1800$ Vevey (Switzerland)

E-Mail skaria@vtxnet.ch 


\section{References}

1 Fiechter S, Skaria A, Nievergelt H, Anex R, Borradori L, Parmentier L: Facial basal cell carcinomas recurring after photodynamic therapy: a retrospective analysis of histological subtypes. Dermatology 2012;224: 346-351.

2 Lomas A, Leonardi-Bee J, Bath-Hextall F: A systematic review of worldwide incidence of nonmelanoma skin cancer. Br J Dermatol 2012; 166:1069-1080.
3 Birch-Johansen F, Jensen A, Mortensen L, Olesen AB, Kjær SK: Trends in the incidence of nonmelanoma skin cancer in Denmark 1978-2007: rapid incidence increase among young Danish women. Int J Cancer 2010;127:2190-2198.

-4 Huber A, Huber JD, Skinner RB Jr, Kuwahara RT, Haque R, Amonette RA: Topical imiquimod treatment for nodular basal cell carcinomas: an open-label series. Dermatol Surg 2004;30:429-430.

5 Schiessl C, Wolber C, Tauber M, Offner F, Strohal R: Treatment of all basal cell carcinoma variants including large and high-risk lesions with $5 \%$ imiquimod cream: histological and clinical changes, outcome, and follow-up. J Drugs Dermatol 2007;6:507-513. 